\title{
Longitudinal stress analyses of columns constrained by rebar under local compression
}

\author{
Y. Wang \\ College of Engineering and Technology, Jilin Agricultural University, Changchun, Jilin, China
}

Keywords: Local compression; plain concrete columns; rebar meshes; longitudinal stress; finite element analysis

\begin{abstract}
Simulations of local compression on the centers of plain concrete square columns with rebar meshes and plain concrete square columns were conducted by means of the finite element analysis software ANSYS. The results show the distributions of longitudinal stress is basically consistent between the plain concrete column and the plain concrete columns equipped with rebar meshes. Increased stress concentration phenomena of load bearing surface, with the load increases. Local compression area ratio affect the rate of diffusion of longitudinal stress, In the vicinity of the pressure surface, local compression area ratio increases, the peak stress in-creased considerably, and local compression bearing capacity is reduced.
\end{abstract}

\section{Introduction}

In the project, deal with the problem of concrete local compression is usually using steel mesh in local compression member ends to enhance the compressive strength of concrete, limiting concentration may occur cracks at the ends. But in the concrete structure, engineering accidents arising because of longitudinal cracks or insufficient bearing capacity are common.

Therefore, this paper by using nonlinear finite element analysis on 3 models with local compression on the center of the plain concrete square columns with rebar meshes to study the failure mechanism of local compression of the concrete.

\section{Methodology}

\section{Material simulation}

Concrete was meshed with SOLID65, which is a type of three-dimensional solid element with eight nodes. Loading plates were meshed by SOLID45 (Barbosa,A.F.\&Ribeiro,G.O.1998), which is another type of three-dimensional solid element with eight nodes. LINK10 elements were adopted to discrete rebar meshes. The constitutive law of concrete was chosen to be isotropic elastoplastic with Von Mises yield criterion and Multilinear Isotropic Hardening Plasticity, which is widely used for proportional loadings and finite deformations. The steel loading plates' material was set to be isotropic linear elastic(Cook, R. D. 2002; Douglas, M.R. \& Lunniss, R.C.1970; Hrabok, M.M.1981; Isenberg, J.1991; Logan, D. L.2002). The concrete has an elastic modulus of $21641 \mathrm{Mpa}$ and a Poisson's ratio of 0.2 . Both the steel loading plates and rebar meshes have an elastic modulus of 210000Mpa and a Poisson's ratio of 0.3. Area of reinforcement is 28.26.

\section{Establishment and Solution of Finite Element Model}

The simulation specimens are concrete square columns of the size $520 \times 300 \times 300$. The loading plates' sizes are, respectively, $165 \times 165 \times 20,80 \times 80 \times 20$, and $40 \times 40 \times 20$.

In the simulations, because all of the columns were symmetric structures locally compressed at their centers by symmetric loads, only $1 / 4$ of each model was simulated and analyzed. Assume that the axis of symmetry is the z-axis, the intersection of the $\mathrm{z}$-axis and the top surface of the column is the origin $\mathrm{o}$, and the top surface of the column is the xoy plane where $\mathrm{x}$ is horizontal and $\mathrm{y}$ is vertical.

A rebar mesh consists of ten $\Phi 6$ rebars, in which five are laid in the ox direction, and the other five are in the oy direction. Five rebar meshes were installed in each column. The top rebar mesh is $30 \mathrm{~mm}$ from the top surface of the concrete column, and the distance between neighboring meshes is 
$50 \mathrm{~mm}$, as shown in Fig. 1- in which Fig. 1a, b, and c are respectively the finite element models for the plain concrete columns embedded with rebar meshes with local compression areas $165 \times 165$ (numbered as W-165), 80×80 (numbered as W-80), and 40×40 (numbered as W-40).

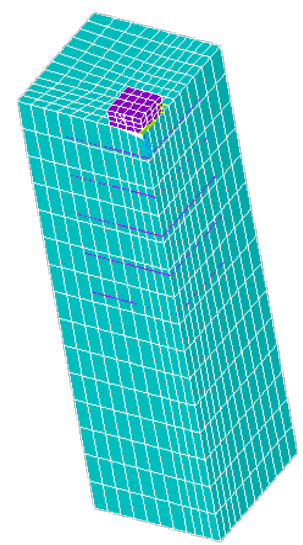

(a)W-165

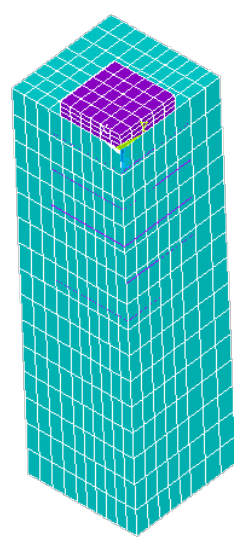

(b) W-80

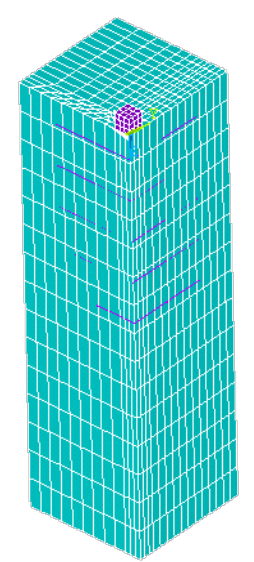

(C) W-40

Figure 1. Finite element models

In each finite element model, constraints of symmetry are applied to both the concrete cutting surface and the loading plate cutting surface, and translational degrees of freedom in all three directions at the nodes on the bottom surface are fixed. In the process of nonlinearly solving each model, the applied load is $500 \mathrm{~N} / \mathrm{mm} 2$, and the step is divided into 100 increments.

\section{Result}

\section{Longitudinal stress distribution}

Figures 2 through 4 demonstrate the results of the finite element simulations of the plain concrete columns with rebar meshes W-165, W-80, and W-40 under local compression where Fig. a, b, and c illustrate the distributions of longitudinal stress in the initial, intermediate, and final phases of loading.

Distributions of longitudinal stress $\sigma \mathrm{x}(\sigma \mathrm{y})$ on the xoz (yoz) plane in plain concrete columns with rebar meshes

From Fig. 2 (a), (b) to (c), can see that, the concrete just below local compression loading position is suffered large stress, and stress decreases to around, when out of range of local compression loading position, stress decreases rapidly, later, the compressive stress decreases gradually, peak compressive stress appears at the bottom of the concrete of local compression loading position, since the compressive stress is gradually reduced to the surrounding, the stress gradually approaches zero at the edges. At a little distance from the load position, the compressive stress on the cross section gradually decreases from the center to the circumference. In the Local compression loading position, longitudinal compressive stress of concrete column is larger, and 
decreases in the longitudinal direction. In the more distant from the load position compressive, stress tends to be uniform, increased stress concentration phenomena of load bearing surface, with the load increases. W-165 compared with the same size plain concrete structures, the position of the peak compressive stress component has not changed, but the compressive stress smaller, local compression bearing capacity increased.

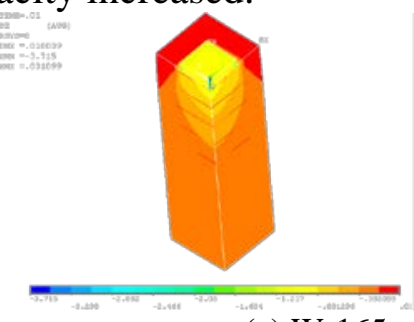

(a) W-165

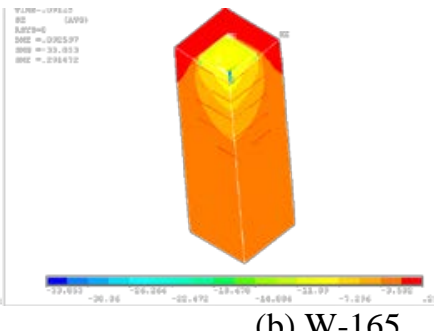

(b) W-165

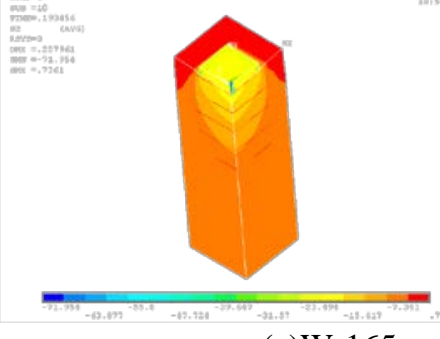

(c)W-165

Figure 2. W-165 Longitudinal stress distribution

From Fig. 3 (a), (b) to (c), can see that, the longitudinal stress distribution of W-80 is similar to $\mathrm{W}-165$, near the local compression surface stress concentration increases with the increase of load; compared W-80 with the W-165, W-80's stress concentration leveled off earlier , and the local compression bearing capacity is reduced; W-80 compared with the same size plain concrete structures, the position of the peak compressive stress component has not changed, but the compressive stress smaller, local compression bearing capacity increased.

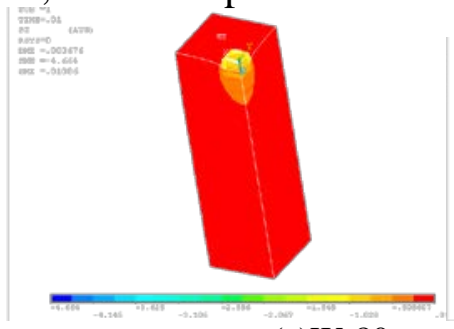

(a)W-80

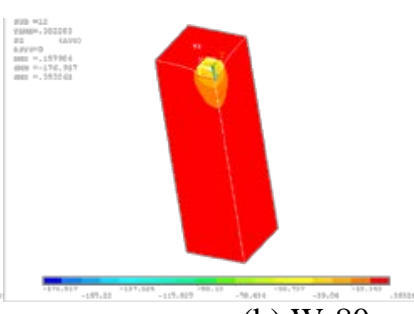

(b) W-80

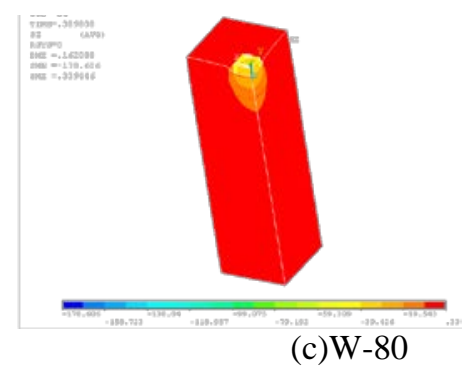

Figure 3. W-80 Longitudinal stress distribution

From Fig. 4 (a), (b) to (c), can see that, the longitudinal stress distribution of W-40 is similar to W-165 and W-80, near the local compression surface ,stress concentration increases with the increase of load, compared W-40 with W-165 and W-80, W-40's stress concentration leveled off earlier, and the local compression bearing capacity is reduced. W-40 compared with the same size plain concrete structures, the position of the peak compressive stress component has not changed, but the compressive stress smaller, local compression bearing capacity increased. 


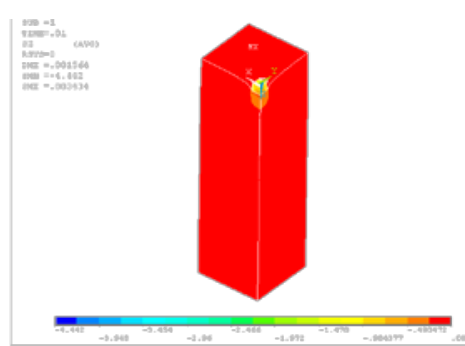

(a) W-40

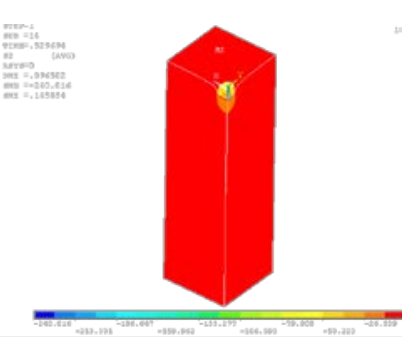

(b) W-40

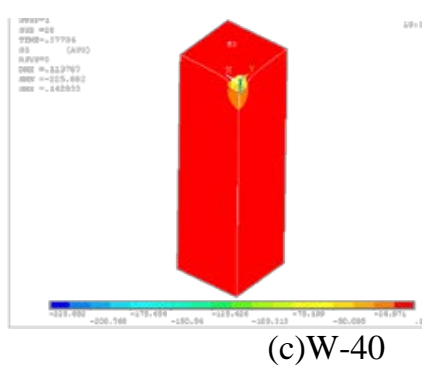

Figure 4. W-40 Longitudinal stress distribution

\section{Conclusions}

The distribution of longitudinal stress is basically consistent between the plain concrete column and the plain concrete column equipped with rebar meshes. Near the local compression surface, stress concentration increases with the increase of load. Local compression area ratio affect the rate of diffusion of longitudinal stress, In the vicinity of the pressure surface, local compression area ratio increases, the peak stress increased considerably, and local compression bearing capacity is reduced.

\section{Acknowledgment}

This work supported by the 12th Five-Year Plan of Jilin Provincial Education Development (Grant No.jijiaokehezi2013di71).

\section{Reference}

[1] Barbosa, A. F., and Ribeiro, G. O., Analysis of Reinforced Concrete Structures using ANSYS Nonlinear Concrete Model, Computational Mechanics, CIMNE, Barcelona, Spain, 1998, pp. 1-7.

[2] Cook, R. D. e. a., CONCEPTS AND APPLICATIONS OF FINITE ELEMENT ANALYSIS. New York: John Wiley \& Sons, Inc., fourth ed., 2002.

[3] Douglas, M. R. and Lunniss, R. C., “An Application of the Finite Element Technique in Bridge Design,” Concrete, vol. 4, pp. 197-200, May 1970.

[4] Hrabok, M. M., Stiffened Plate Analysis by the Hybrid Stress Finite Element Method. PhD thesis, University of Alberta, Edmonton, Alberta, Canada, 1981.

[5] Isenberg, J., ed., FINITE ELEMENT ANALYSIS OF REINFORCED CONCRETE STRUCTURES II, (New York, New York), Committee on Finite Element Analysis of Reinforced Concrete Structures, American Society of Civil Engineers, June 1991.

[6] Logan, D. L., A FIRST COURSE IN THE FINITE ELEMENT METHOD. Pacific Grove, CA: Brooks/Cole, third ed., 2002. 